\title{
Classifying Urban Building from Road Based on Fuzzy Logic Rules and Climate Proofing Codes Using Quick Bird Imagery
}

\author{
Li Hongfen, Hu Guangdao, Du Jun, and Wang Chao
}

\begin{abstract}
This article deal with the problem of distinguishing buildings from roads of land cover maps using very high-resolution satellites (VHRS). It discusses two methods for improving the result of classifying these two land use types in land cover mapping. A case study of QuickBird imagery of an area in Chenggong County of Yunnan Province is conducted based on an analysis of QuickBird imagery.
\end{abstract}

Index Terms-Fuzzy logic system (FLS), spatial feature extraction, texture analysis, image segmentation.

\section{INTRODUCTION}

\section{A. Background}

Classification of land-cover information using high-resolution remotely-sensed imagery is a challenging topic due to the complexity of landscapes and the spatial and spectral resolution of the images being used. High-resolution remote sensing images have many more spatial and textural characteristics than low-resolution data except spectral characteristics, but also make great challenge to current classification methods. As the resolution gets finer, the landscapes show more complicated spatial characteristics in the image, more noise information show up apart from the patterns we really want to extract.

Recently, numerous studies have applied artificial intelligence sensed image classification applications. There is a major drawback in applying these models that the user cannot readily realize the final rules. How to design an effective classification method, which can handle these problems and make full use of both spatial and spectral features, becomes a fundamental research topic in the field of high resolution imagery classification.

\section{B. Climate Proofing Codes}

Extreme weather events and changing climate can wreak havoc on properties and could potentially have a devastating effect on environment. The purpose of Climate-Proofing is to present available, unique, and sometimes surprising products that can be used to protect communities and homes from the

Manuscript received November 11, 2014; revised January 10, 2015.

Li Hongfen is with the Institute of Geographical Sciences, Henan Academy of Sciences and China University of Geosciences, Resources Academy, China (e-mail: lihongfen_dida@163.com).

$\mathrm{Hu}$ Guangdao is with the China University of Geosciences, Resources Academy, China (e-mail: gdhu@cug.edu.cn).

Du Jun and Wang Chao are with the Institute of Geographical Sciences, Henan Academy of Sciences, China (e-mail: Dujun2008@163.com, 1208483461@qq.com). ravages of extreme weather events worldwide. Last several years, Chenggong County of Yunnan Province has suffered an extraordinary string of extreme climate events and cost huge damage. So nowtime, it is very important to have a map indicated the intensity of environment stress geographyically, which makes it clear which part of district is in danger depending on the monitoring of environment issues. And as the climate continues to change, we are able to evaluate the vulnerablity against climate disasters.

As in research [1], changes in land cover inlude changes in biotic diversitym actual and actual and ptential primary productivity, soil quality, runoff, and sedimentation rates, and cannot be well understood without the knowledge of land use change that drives them. Therefore, land use and land cover changes have environmental implications. Researchers found a huge challenge of defending the change of a hot climate. The climate problem of UHI (Urban Heat Islands) showed, urban areas where the air could be hotter than its surrounding areas and where pollution levels are consequently raised.

Buildings is the very symbol of urban city .By locating and maping of buildings, we can monitor and analysis the intensity of urban expanding. In this paper we build a method of classification by extract 2 spectral features and 4 geographic features from Remote Sensing images, to classify buildings from other Geographical entity and comparison the result.

\section{AIMS AND STRUCTURE OF THIS RESEARCH}

We focus on dealing with the misclassification problem of building and road using QuickBird Imagery. According to the previous researches [2], [3], these two are often misclassified to each other because they have very similar spectral characteristics on high resolution imagery, and road itself can be divided into multiple kinds according to their spectral differences. How to classify these two kinds of landscape which have very similar spectral features?

We notice that although these two have very similar, or almost the same spectral features, they are very different in shape. So, in this paper, Instead of focusing exclusively on the spectral information contained in the data, we present a classification procedure in which spatial patterns can be used when classifying, such as shape, length, the ratio of length/width, etc, apart from spectral features. Also, fuzzy logic rule-based classifier (FLS for short) is used to handle the problem of distinguishing very close or overlapped classes.

The data used in this paper were acquired over an agricultural area near the village of Chenggong district in Yunnan province, and was collected in 2003(QuickBird). The 
image area is about $780^{*} 600$ pixels, located in E102 $00^{\prime} 04^{\prime \prime} \sim 102^{\circ} 09^{\prime} 44^{\prime \prime}, \mathrm{N} 24^{\circ} 05^{\prime} 25^{\prime \prime} \sim 24^{\circ} 05^{\prime} 37^{\prime \prime}$. The data contains red, green, blue and NIR bands, with an additional panchromatic band. After fusion, the resolution of the image was improved to $0.61 \mathrm{~m}$. Before classification; some pre-processing work was done. Geometric correction is performed using 25 GCPs (Ground Control Point); PCA was applied for spectral enhancement; orthorectification of the image is done with 40 GCPs using a Polynomial model, whose RMS is $1.93 \mathrm{~m}$. Atmospheric correction was made to the image using Dark-Object-Methods. The same area was processed by every classification test, in order to assess the ability of classifying of these methods. The same training set was used, also a test set for accuracy assessment.

\section{METHOD}

\section{A. Normal Difference Vegetation Index (NDVI) Analysis}

Vegetation indices (VIs) are combinations of surface reflectance at two or more wavelengths designed to highlight a particular property of vegetation. They are derived using the reflectance properties of vegetation. The normalized difference vegetation index (NDVI) is one of the oldest, most well known, and most frequently used VIs.

Considering that this research focus mainly on the distinguishing of buildings and roads, which have distinct difference of $\mathrm{DN}$ (digital number) values from other landscapes such as vegetations and water in an NDVI image, we excluded water and vegetation area out of the research area by building multiple fuzzy logic rules using NDVI image. NDVI is defined by Equation 1:

$$
N D V I=\frac{\rho_{N I R}-\rho_{R E D}}{\rho_{N I R}+\rho_{R E D}}
$$

where $\rho_{\text {nir }}$ and $\rho_{\text {RED }}$ are the NIR and RED band of the image.

\section{B. Image Segmentation}

Various segmentation algorithms have been proposed in the literature with promising results. The choice of which kind of segmentation algorithms to be included in the image analysis system is up to the particular aim of the system and the problems it has to deal with. In this paper, Firstly, an edge-based segmentation algorithm was used to cluster the adjacent pixels into geographic objects. By suppressing weak edges to different levels, multi-scale segmentation results from finer to coarser segmentation were produced. By controlling the segmentation levels, appropriate size of objects were created according to the size of landscape types in the real world in case of over segmentation.

At the same time, another problem showed up, because the objects belonging to the same landscape types have a changing range of sizes, segmentation cannot make every object integrate, a redundancy of polygons was produced. These redundancies can also lead to misclassification because it corrupts the integrity of objects which contain the distinguishing spatial features of real-world landscapes, as the spatial feature we built are based on geographically integrate objects rather than segments of them. So we have to merge the adjacent segments which have similar spectral and spatial features into geographically reasonable objects. In this paper, we use an algorithm iteratively merges adjacent segments based on both spectral and spatial information, as in research [4], Robinson, Redding and Crisp used this algorithm originally for segmentation of SAR imagery. If the algorithm finds a pair of adjacent regions, $i$ and $j$, such that the merging $\operatorname{cost} t_{i, j}$ is less than a defined threshold value, it is expressed in Equation 2:

$$
t_{i, j}=\frac{\left|O_{i}\right| \cdot\left|O_{j}\right|}{\left|O_{i}\right|+\left|O_{j}\right|} \cdot \| u_{i}-u_{j}||
$$

where $O_{i}$ is region $i$ of the image; $\left|O_{i}\right|$ is the area of region $i$; $u_{i}$ is the average value in region $i ; u_{j}$ is the average value in region $j ;\left\|u_{i}-u_{j}\right\|$ is the Euclidean distance between the spectral values of regions $i$ and $j$; length $\left(\partial\left(O_{i}, O_{j}\right)\right)$ is the length of the common boundary of $O_{i}$ and $O_{j}$.

\section{Spatial Feature Extraction}

Spatial features are very important during classifying when two classes are very close or even overlapped spectrally. Whether the rules can distinguish the landscape types correctly or not lies on the appropriate choice of spatial features. In our research area, the DN values of most road and building are almost the same, which indicate that they are built of very similar material, make it harder to classify these two. And, the fact is, there are two kinds of roads which are made of different material, and they show very different spectral features.

So here we try to build the following 3 main features for the classifying:

1) ELONGATION, A shape measure which indicates the ratio of the major axis of the polygon to the minor axis of the polygon. The max and minor axes are derived from an oriented bounding box containing the polygon. ELONGATION = MAXAXISLEN / MINAXISLEN, The elongation value for a square is 1.0 , and the value for a rectangle is greater than 1.0.

2) LENGTH, The combined length of all boundaries of the polygon, including the boundaries of the holes.

3) RECT_FIT, A shape measure that indicates how well the shape is described by a rectangle. This attribute compares the area of the polygon to the area of the oriented bounding box enclosing the polygon. The rectangular fit value for a rectangle is 1.0 , and the value for a non-rectangular shape is less than 1.0.

\section{RECT_FIT $=$ AREA/(MAXAXISLEN $*$ MINAXISLEN)}

where AREA is the total area of the polygon, minus the area of the holes; MAJAXISLEN is the length of the major axis of an oriented bounding box enclosing the polygon; MINAXISLEN is the length of the minor axis of an oriented bounding box enclosing the polygon. Values are map units of the pixel size. 
After merging adjacent segments, we found that some redundancy segments still remain, so we use a texture segmentation method to confine the integrity of the polygons and get more geographically reasonable objects. The result was used to assistant the classification process as an additional band to the original image.

Firstly, an edge-based detection was applied; secondly, a PCA (Principle Component Analysis) transformation was applied to the edge-based detection result image; thirdly, a low-pass filter was used and then segmented; finally, a texture-enhanced binary-value image was produced.

Combine the result image with the original image, and use them in the segmentation process. In this way, more effective rules can be created to distinguish classes with fuzzy logic rule-based classifier.

According to the previous researches [5], [6], we choose two other texture features into calculation:

$$
\begin{gathered}
\text { Mean: } f_{M E A}=\sum_{i=0}^{M} \sum_{j=0}^{N} i \times p(i, j) \\
\text { Variance: } f_{V A R}=\sum_{i=0}^{M} \sum_{j=0}^{N} p(i, j) \times(i-u)^{2}
\end{gathered}
$$

where $u=\sum_{i=0}^{M} \sum_{j=0}^{N} i \times p(i, j), p(i, j)$ is the DN(Digital

Number) value of the pixel that locate in Line $i$, Row $j$ of the test image $(M \times N)$.

\section{Fuzzy Logic Systems (FLS)}

Information extraction from remote sensing data is limited by noisy sensor measurements with limited spectral and spatial resolution, signal degradation from image pre-processing, and imprecise transitions between land-use classes. Most remote sensing images contain mixed pixels that belong to one or more classes. Fuzzy logic helps alleviate this problem by simulating uncertainty or partial information that is consistent with human reasoning.

The expression of environmental features is often hampered by the fact that their definition is vague. The definitions of vagueness concepts means: the features can only be identified with a limited level of certainty so that there is a substantial fuzziness in their spatial description..As in Research [7], FLS is famous for dealing with uncertainties and imprecision.

Traditional rule-based classifier is based on strict binary rules. Rather than saying that something is fully "true" or false" (as in binary rules), FLS uses membership functions to represent the degree than an object belongs to a feature type. Information extraction from remote sensing data is limited by noisy sensor measurements with limited spectral and spatial resolution, signal degradation from image pre-processing, and imprecise transitions between land-use classes. Most remote sensing images contain mixed pixels that belong to one or more classes. Fuzzy logic helps alleviate this problem by simulating uncertainty or partial information that is consistent with human reasoning.

The spatial extent of geo-objects is generally determined through the boundaries, or more precisely, through the position of the boundary points. The analysis of the geometric uncertainty of the objects is therefore often based on accuracy models for the co-ordinates of these points. Because it is not only a matter of co-ordinate accuracy; it is rather a problem of object definition and thematic vagueness. This becomes apparent when mapping is based on feature extraction from digital images with a raster structure rather than with a vector structure commonly used in land surveying and photogrammetry.

\section{EXPERIMENTS}

\section{A. Researching Area and Image Pre-processing}

The data used in this paper were acquired over an agricultural area near the village of Chenggong district in Yunnan province, and was collected in 2003(QuickBird). located in E102 $00^{\prime} 04^{\prime \prime} \sim 102^{\circ} 09^{\prime} 44^{\prime \prime}$, N24 $4^{\circ} 05^{\prime} 25^{\prime \prime} \sim 24^{\circ} 05^{\prime} 37^{\prime \prime}$. The data contains red, green, blue and NIR bands, with an additional panchromatic band. After fusion, the resolution of the image was improved to $0.61 \mathrm{~m}$. Before classification; some pre-processing work was done. Geometric correction is performed using 25 GCPs (Ground Control Point); Porthorectification of the image is done with 40 GCPs using a Polynomial model, whose RMS is $1.93 \mathrm{~m}$. Atmospheric correction was made to the image using Dark-Object-Methods. The same area was processed by every classification test, in order to assess the ability of classifying of these methods. The same training set was used, also a test set for accuracy assessment (Fig. 1)

\section{B. Flow of Research}

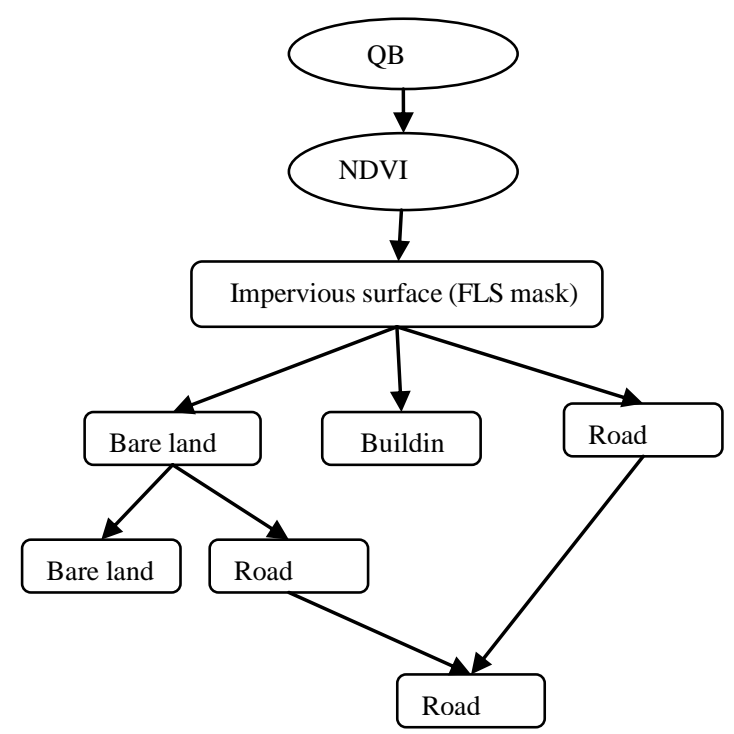

Fig. 1. The work flow of the research.

\section{Result Analysis}

Part of the results in this study are showed in Fig. 2; Fig. 2(a) shows the original image of study area; Fig. 2(b) is the NDVI calculation result of original image; Fig. 2(c) shows the results after texture segmentation was applied on NDVI image; Fig. 2(d) is the texture feature results of Mean over NDVI image with window size of $3 \times 3$ pixels; Fig. $2(\mathrm{e})$ is the FLS confidence image with the confidence of 0.7; Fig. 2(f) shows the result image after we applied Fig. 2(e) as a mask 
over original image.

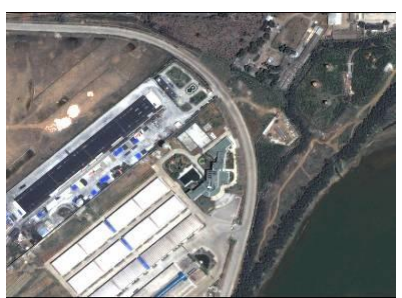

(a) Original QuickBird image

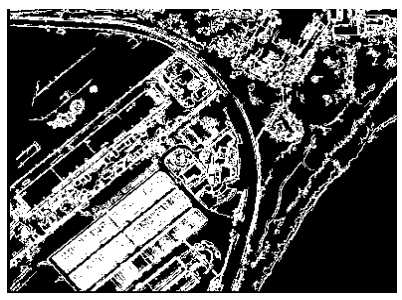

(c) Texture segmentation image

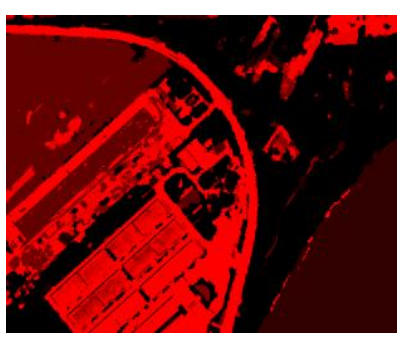

(d) Texture Mean image $(3 \times 3)$

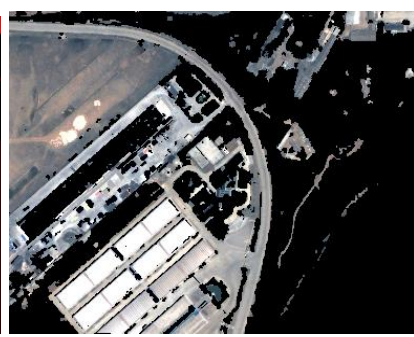

FLS rule confidence image (0.7) (f) Mask image of building and road

Fig. 2. A list of results from every step according to the work flow.

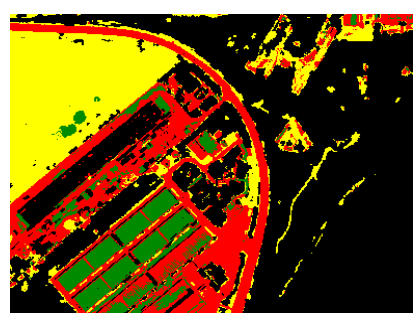

(a) SVM with original 4 bands

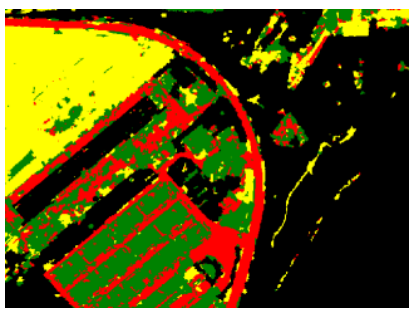

(c) FLS with texture enhance

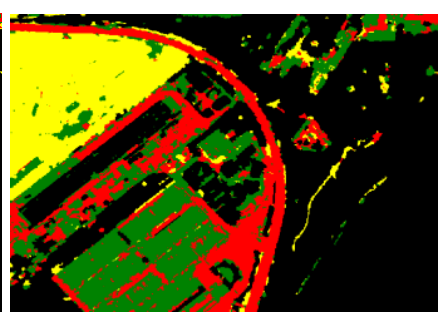

(b) FLS with original 4 bands

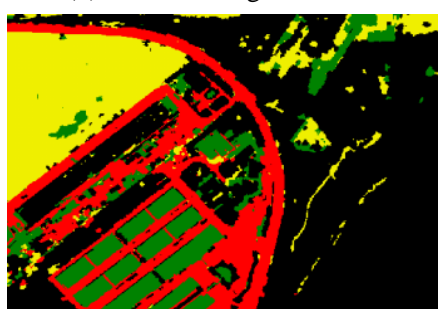

(d) FLS with spatial \& texture features

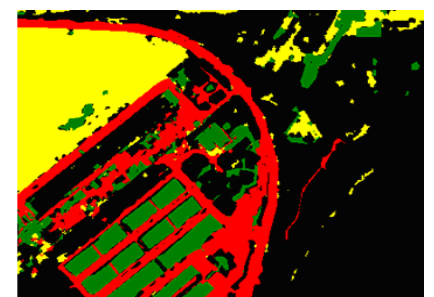

(e) Final classification results with FLS and spatial and texture features

Fig. 3. The classification results of different classifiers with or without spatial\& texture features.

In order to assess the ability of classifying, we choose SVM (Support Vector Machine) to perform classification with the same training set of data, and the result was taken as a comparison to FLS.

According to the research [8], SVM were developed by
Vapnik in 1995. Instead of applying empirical risk minimization (ERM) principle commonly used in the statistical learning methods, SVM employ structural risk minimization (SRM) principle to achieve better generalization ability. It is very suitable at dealing with high-dimensioned data and can get satisfactory result while classifying classes that are very close in feature space.

In this section, 4 tests were made with different classifiers and feature combinations to assess their contribution to the promotion of classifying accuracies. Fig. 3 shows the test results. Red area represents Road type1, Green means buildings, and yellow is Spaceland. These 3 have different DN values on the image. While Road type 2 has the same spectral features as Bare land, it will be extracted separately.

Fig. 3(a) to Fig. 3(d) shows the results of 4 different situations: classification using SVM, FLS with original 4 bands, FLS with ONLY texture enhancement, FLS with spatial/texture features and texture enhancement. Compare Fig. 3(b) and Fig. 3(c), we make another mask and classify Road type 2 from Bare land by building another set of fuzzy logic rules. Considering the linear shape of road2, it can be easily extracted from Bare land. Fig. 3(f) shows the final result after combining Road type2 to Road type1.

\section{Accuracy Analysis}

To assess the performance of these classifiers, accuracy was estimated using the same training samples collected for land cover mapping. The results in Table I show that FLS produced substantial increases in estimated accuracy when compared to SVM classifier. Adding spatial and texture features into classification with FLS classifier gets the highest accuracy of $92.2039 \%$, the kappa is 0.8438 . Adding a texture band to the original image with FLS classifier makes an improvement of $4.617 \%$, compared to using only the original 4-band image. It gets the accuracy of $90.8838 \%$, which is the second highest.

Table I to Table II show the confusion matrixes and accuracy assessment of Fuzzy logic rule-based classification results, in which spatial and texture features were included to help classify. Table III is a comparison result of original image classification (only contain 4 bands: R, G, B, N) with Fuzzy logic classifier, and shows a difference in accuracy with or without the spatial/texture features.

TABLE I: CONFUSION MATRIXES OF FUZZY LOGIC RULE CLASSIFICATION WITH SPATIAL AND TEXTURE FEATURES

\begin{tabular}{|c|c|c|c|}
\hline Class(pixels) & road & building & Total \\
\hline Road1 & 9241 & 352 & 9593 \\
\hline building & 1116 & 8121 & 9237 \\
\hline total & 10357 & 8473 & 18830 \\
\hline
\end{tabular}

TABLE II: CLASSIFICATION ACCURACY

\begin{tabular}{|l|l|l|l|l|}
\hline class & $\begin{array}{l}\text { Commission } \\
(\%)\end{array}$ & $\begin{array}{l}\text { Omission } \\
(\%)\end{array}$ & $\begin{array}{l}\text { Prod. Acc } \\
(\%)\end{array}$ & $\begin{array}{l}\text { User. Acc } \\
(\%)\end{array}$ \\
\hline Road1 & 3.67 & 10.78 & 89.22 & 96.33 \\
\hline Building & 12.08 & 4.15 & 95.85 & 87.92 \\
\hline
\end{tabular}

From the accuracy results, we can see that FLS gets an accuracy of $86.2668 \%$, while SVM is $81.2782 \%$ using only spectral features. It shows that FLS is superior over SVM at separating these two classes (road and building) which are very close or even overlapped to each other. The inclusion of texture segmentation band improves the accuracy to 
$90.8838 \%$ with FLS, which makes a further improvement to $91.9992 \%$ by adding spatial and texture features. The adding of spatial and texture features makes an increase of $4.617 \%$ and the FLS makes an increase of $4.9886 \%$ over SVM.

TABLE III: THE ACCURACY COMPARISON OF SEVERAL COMBINATIONS OF TEXTURE BAND AND CLASSIFIER

\begin{tabular}{|l|l|l|l|l|}
\multicolumn{2}{c}{ TABLE III: THE ACCURACY COMPARISON OF SEVERAL COMBINATIONS OF TEXTURE BAND AND CLASSIFIER } \\
\hline & 4 original bands (SVM) & 4 original bands (FLS) & Adding texture enhance (FLS) & $\begin{array}{l}\text { Texture-enhance \& spatial \& texture } \\
\text { (FLS) }\end{array}$ \\
\hline Overall accuracy (\%) & 81.2782 & 86.2668 & 90.8838 & 92.2039 \\
\hline kappa & 0.5272 & 0.7192 & 0.8165 & 0.8438 \\
\hline Road1 (\%) & 79.06 & 87.96 & 88.41 & 96.33 \\
\hline Building $(\%)$ & 91.31 & 83.99 & 94.08 & 87.92 \\
\hline
\end{tabular}

It shows the applying of spatial and texture features and FLS make distinct improvement to classification accuracy. Also, we can find that although the FLS result (with spatial and texture features) gets the highest overall accuracy, it does not get the highest for every particular class. For example, buildings get a decline in accuracy after adding spatial features compared to only texture-enhanced image. Meanwhile, we also noticed that SVM gives a better result at classifying buildings over FLS, although FLS gets a higher overall accuracy. There is a possibility that every classifier has its predominance at classifying some particular classes, as in this paper, FLS is not good at classifying buildings. It gives us a hint that MCS (Multiple Classifier Systems) can be added to the subsequent research to improve the classifying abilities ulteriorly.

\section{CONCLUSIONS}

In this paper, an approach to the automatic design of FLS formed by different classification rules based on spatial and spectral features has been described. The comparison results shows (in Table III) that the inclusion of texture segmentation band improves the accuracy to $90.8838 \%$ with an increase of $4.617 \%$ ० The applying of FLS makes a further improvement to $91.9992 \%$ by adding spatial and texture features, which gets an increase of $5.9371 \%$. FLS makes an improvement of accuracy of $4.9886 \%$ over SVM using the same original image. However, not every particular class benefit from FLS, it means FLS can only improve the overall accuracy, but not good at classifying every class. As to feature extraction, the accuracy assessment shows that the adding of spatial features (especially shape and texture factor) can improve the result greatly.

But we also notice that, although most roads can be extracted from building using this method, some misclassification still remained. For example when roads are interrupted by tree shades, grass etc., integrate road shape features can not be extracted, therefore the classification are not reliable depending on this linear feature of road. Also, most of time, buildings and space land have the same special and spectral features. If objects of them with the same spectral and spatial features were created, the method described in this paper cannot classify correctly. In the future, context-sensitive method should be considered to deal with this problem, for example, buildings always have shadows adjacent to them due to the attribute of height. Then we can make rules define buildings as they have shadows adjacent to them.

\section{ACKNOWLEDGMENT}

First, I would like to express my sincere gratitude to all those who helped me during the writing of this thesis.

Second, I would like to extend my heartfelt gratitude to my supervisor, Pro. Hu Guangdao for his instructive advice on my thesis, and also those people, without his consistent and illuminating instruction, I could not finish this thesis.

Finally, I would like to thank the Institute of geography of Henan Academy for providing the image data.

\section{REFERENCES}

[1] Q. Weng, “A remote sensing-GIS evaluation of urban expansion and its impact on surface temperature in the Zhujiang Delta, China," In. J. Remote Sensing, vol. 22, pp. 1999-2014, 2001.

[2] S. Chen, B. Mulgrew, and P. M. Grant, "A clustering technique for digital communications channel equalization using radial basis function networks," IEEE Trans. on Neural Networks, vol. 4, pp. 570-578, July 1993.

[3] M. Young, "Segmentation and object-based classification for the extraction of the building class from LIDAR DEMs computers \& geosciences," in The Technical Writer's Handbook, G. Miliaresis and N. Kokkas, Eds. Mill Valley, CA: University Science, vol. 33, pp. 1076-1087, 2007.

[4] L. Durieux, E. Lagabrielle, and A. Nelson, "A method for monitoring building construction in urban sprawl areas using objected-based anaysis of Spot 5 image and existing GIS data," Photogrammetry \& Remote Sensing, vol. 63, pp. 399-408, 2008.

[5] D. J. Robinson, N. J. Redding, and D. J. Crisp, "Implementation of a fast algorithm for segmenting SAR imagery," Scientific and Technical Report. Australia: Defense Science and Technology Organization, January 1, 2002.

[6] R. M. Haralick, K. Shanmugam, and I. Dinstein, "Texture features for image classification," IEEE Transactions on Systems, Man, and Cybernetics, vol. 3, pp. 610-625, 1973.

[7] S. Berberoglu, P. J. Curran, C. D. Lloyd, and P. M. Atkinson, "Texture classification of mediterranean land cover," International Journal of Applied Earth Observation and Geo-information, vol. 9, pp. 322-334, 2007.

[8] G. Miliaresis and N. Kokkas, "Segmentation and object-based classification for the extraction of the building class from LIDAR DEMs," Computers \& Geosciences, vol. 33, pp. 1076-1087, 2007.

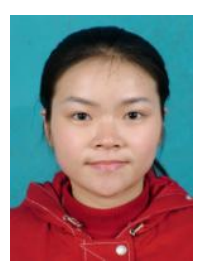

Li Hongfen was born in Wuhan, Hubei Province of China. In 2000, after finishing college course in China University of Geosciences, she was sent to Beijing for post-graduate study, she owned the bachelor degree in the Resources Department. After researching in the Institute of Geology and Geothysics, Chinese Academy of Sciences, she owned the master degree of engineering in the Physical Geography Department, majored in earth detect and information technology. In 2013, she became a PhD student in the Institute of Mathematics Geology and Remote Sensing Geology, in the research field of environmental monitoring of remote sensing. In 2014, after one year's lessons of theory, she turned to the Institute of Geography of Henan Province for further research on RS technology in environmental monitoring.

She has several academical dissertations published on the core journals in Chinese as the first authors and an English dissertation titled "Multi-clisifier system (MCSs) of remote sensing imagery classification based on texture analysis" was published by Springer on Lecture Notes of Computer Science in 2012 . 